Results Only eight articles, amongst them involving 166 patients, met the study criteria. Themes demonstrated repeated hospital admissions towards the end of life, lack of coordinated care in community and barriers in discussion about palliative care in end stage liver disease due to lack of confidence amongst professionals and a negative view about palliative care amongst patients and carers. Emotional, financial and disability related needs of patients and their carers are often neglected. (Table 1)

Conclusions Further research into perspectives of patients with advanced liver disease and their carers regarding palliative and EOL care will help improve the implementation of patientfocussed palliative care, advance care planning and EOL care. Research should also be done into the barriers to EOL discussions so that they can be held earlier with the aim of improving patient and carer quality of life and outcomes. It would be interesting to see the comparison of the attitude to, and expectations from, palliative care among patients and their caregivers from different parts of the world where the aetiological spectrum of liver disease, the social construct of healthcare and availability of palliative care differs significantly.

\section{PTH-49 SUCCESSFUL ENDOSCOPY RECOVERY STRATEGY AFTER THE FIRST WAVE OF THE COVID-19 PANDEMIC}

Paolo Giuffrida*, lain Ewing, Eleanor Wood, Nora Thoua, Henning Spranger, Jun Liong Chin, Esra Asilmaz, Ray Shidrawi, Laura Marelli. Homerton University Hospital, London, UK

\subsection{6/gutjnl-2021-BSG.364}

Introduction Endoscopy other than essential or emergency cases was paused in March 2020 during the first wave of the Covid-19 pandemic leading to a significant backlog. In April 2020 the BSG issued new guidance for safe resumption of endoscopy services.

Methods We here described how we managed the endoscopy backlog generated by the first wave of the Covid-19 pandemic in a Covid-minimised unit. We evaluated the impact of service suspension on backlog, recovery strategy, infection control policy, results of pre-procedure Covid-19 testing, and 7/14-day post-procedure Covid-19 symptom screening.

Results 937 elective procedures were cancelled between 23 March and June 2020. A vetting tool linked to the booking system was used to categorise these as High-risk 2-week wait $(n=57)$, Defer 3 months $(n=439)$, Defer 6 months $(n=300)$, Defer 12 months $(n=9)$, Surveillance $(n=45)$, Discharge back to referrer $(n=87)$. Elective procedures restarted on 8 June 2020. Lists were initially booked with $50 \%$ reduction in volume compared to pre-Covid-19, to accommodate PPE, downtime and social distancing. We increased endoscopy administration from 2 to 5 staff, to implement 7-day pre-procedure 'SCOTS criteria' telephone screening, 3-day pre-procedure Covid-19 PCR testing, and 7/14-day post-procedure telephone follow-up. We introduced outpatient information leaflet and consent forms regarding Covid-19 risk. Inpatient endoscopy was carried out in the operating theatre until the end of August. On 17 July we removed downtime after lower GI endoscopy increasing capacity. Twice weekly evening lists resumed in August, with an extra evening list added in September. From 1 August until 10 October we used insourcing at weekend. We trialed outsourcing of 2 weekly lists for 4 weeks in August, but did not find this strategy effective. Additional Saturday and evening lists were performed by 6 endoscopists removed from the GIM rota. We were able to clear our waiting list by mid-October so that we could offer mutual aid to a neighboring hospital.

Between June and November we performed endoscopy in 3,481 outpatients. Each patient had pre-endoscopy Covid-19 swab and $20(0.57 \%)$ were positive. 23 out of $3,261(0.71 \%)$ patients developed Covid-19 symptoms after 7 days and 29 (0.89\%) after 14 days.

Conclusions We demonstrated effective clearance of the endoscopy backlog in a Covid-19 safe environment over 4 months. Key interventions were advance vetting, increased administrative support, an endoscopy unit located in a separate building, quick implementation of infection control policies, insourcing and freeing of endoscopists from the GIM rota. Learning point was underestimating burnout of endoscopy nursing staff.

\section{PTH-50 MICROSCOPIC COLITIS: MIND THE GAP}

${ }^{1}$ Shivani Richards*, ${ }^{2}$ Paul Collins. ${ }^{1}$ The University of Liverpool, Liverpool, UK; ${ }^{2}$ Royal Liverpool University Hospital, Liverpool, UK

\subsection{6/gutjnl-2021-BSG.365}

Introduction Microscopic colitis (MC) is a chronic inflammatory disease that affects the colon. Characteristically it causes chronic, watery, non-bloody diarrhoea, which substantially reduces patient quality of life (QoL). MC may be less familiar than other causes of diarrhoea and could lead to delays in diagnosis of $\mathrm{MC}$, or a failure to diagnose this condition.

Aims 1. To assess the timelines of referral, diagnosis and treatment in patients who are subsequently diagnosed with MC

2. To identify treatments initiated and potential culprit drugs in patients with MC

Methods Patients with MC were identified from electronic health records. Patient demographics including smoking status were gathered. Data was obtained on the date of symptom onset, clinical review, diagnosis and treatment intervention (advice on cessation of a potential culprit drug and/or start of treatment.)

Results 304 patients were diagnosed with MC, 230 females and 74 males (3.1:1). The median age of patients was 69 years (range 19-100). 42\% of patients were current smokers, of which 54\% did not receive smoking cessation advice. 219 patients (92\%) were reviewed by a gastroenterologist postsymptom onset, with a median wait of 251 days (range 17516) from the time of symptom onset. Of those diagnosed with $\mathrm{MC}$ after symptoms, the median wait between symptom onset and diagnosis was 113.5 days (range 1-7286). 57 patients $(25 \%)$ waited $>1$ year and 15 patients $(7 \%)$ waited $>5$ years before diagnosis. For those initiated on treatment post-diagnosis, the median number of days from symptom onset to commencing treatment was 324 days (range 117516). 36 patients had treatment initiated prior to their diagnostic histology report. Most patients (58\%) were given budesonide as their first treatment. $38 \%$ of patients required additional treatments, $17 \%$ required no treatment. Loperamide was given to $46 \%$ of patients receiving additional treatments. $67 \%$ of patients were taking a potential culprit drug, of these $20 \%$ were taking multiple potential culprit drugs (Omeprazole 19.9\%, NSAID 18.7\%, Lansoprazole 15.6\%, Sertraline 8.10\%, Beta blocker $6.54 \%$ ). Of the patients taking a potential culprit drug, 52\% were advised to stop the same drug and $4 \%$ were advised to stop a different drug. 
Conclusion Patients with MC are diagnosed within a median of just over 3 months of symptom onset. Some patients are still referred and diagnosed long after symptom onset. There may be patients with chronic diarrhoea in the community who are undiagnosed. There is some variation in prescribing practice for MC. Standardisation of management pathway in the UK would assist with management (Münch A, et al. Frontline Gastroenterology 2020;11:228-234).

\section{PTH-51 PATIENT SAFETY MATTERS: CARE CLOSER TO HOME IN GASTROENTEROLOGY HIGH COST DRUG MANAGEMENT DURING COVID-19}

${ }^{1}$ Sapna Gohil*, ${ }^{1}$ Arti Soni, ${ }^{1}$ Maimuna Ahmed, ${ }^{1}$ Nikki Lovell, ${ }^{2}$ Duncan Loft, ${ }^{2}$ Esther Unitt, ${ }^{1}$ Hardeep Bagga. 'Pharmacy, University Hospitals Coventry and Warwickshire NHS Trust, Coventry, UK; ${ }^{2}$ Gastroenterology, University Hospitals Coventry and Warwickshire NHS Trust, Coventry, UK

\subsection{6/gutjnl-2021-BSG.366}

Introduction The Covid-19 pandemic has been a strong catalyst in driving the reconfiguration of gastroenterology outpatient models of High Cost Drug (HCD) delivery. This service evaluation aims to explore the impact of multiple quality improvement initiatives synchronously implemented by the HCD pharmacists and the gastroenterology clinical team, to improve medication safety and HCD treatment access.

Methods The Plan-Do-Study-Act (PDSA) quality improvement methodology was applied to three key service areas, from March 2020 to May 2021: i) 95 Hepatitis B (HB) patients receiving FP10 HCD prescriptions were reviewed by the clinical team for switch to the hospital outpatient pharmacy model (HOP), enabling HCD collection from their local pharmacy. Conversely, Hepatitis $\mathrm{C}(\mathrm{HCV})$ patients struggled to reach the HOP. To improve treatment access, 29 eligible HCV patients were offered HCV HCD homecare (HC) delivery, enabling direct delivery to the patient's preferred address ii) development of a digital pharmacy database to record queries and pharmacist interventions supporting safety monitoring for 450 HC gastroenterology patients iii) exploration of alternative HCD formulations available via HC.

Results $100 \%$ of the eligible $\mathrm{HCV}$ patients agreed to the HC model and $100 \%$ of $\mathrm{HB}$ patients agreed to the HOP. HCD pharmacists helped to resolve $114 \mathrm{HC}$ prescription queries in regard to incomplete blueteq proformas, delayed clinic followup reviews and blood monitoring. Subcutaneous (SC) formulations of vedolizumab and infliximab recently became available for self-administration (SA) via $\mathrm{HC}$, as options for eligible inflammatory bowel disease (IBD) patients considered to be at-risk of Covid-19. 41 patients receiving intravenous (IV) vedolizumab at the hospital were eligible for switch to SC via HC. 26/41 patients agreed to switch. 2/26 patients switched back to IV as 1 patient $=$ reported IBD flare and 1 patient $=$ injection site reaction. $15 / 41$ patients refused the switch. IV infliximab patients eligible for switch to SC are currently being reviewed. 72 new starter IBD patients were identified for potential commencement of the new cost-effective adalimumab biosimilar via HC.

Conclusions Gastroenterology HCD prescriptions are now being processed safely via two main outpatient delivery models, both governed by robust contractual arrangements. Improved clinical monitoring has helped to reduce the risk of adverse events, thereby improving medication safety. These simple initiatives also delivered prescribing cost efficiencies in excess of $£ 400,000$ ensuring best use of NHS resources.

\section{PTH-52 PBC MANAGEMENT IN A RURAL SETTING. HOW DO WE COMPARE TO NATIONAL STANDARDS?}

Stewart McDonald* ${ }^{*}$ Eugene Campbell. Western Health and Social Care Trust, Enniskillen, UK

\subsection{6/gutjnl-2021-BSG.367}

Introduction The South West Acute Hospital (SWAH) serves the most rural population in Northern Ireland. The British Society of Gastroenterology (BSG) updated guidelines on the treatment and management of Primary Biliary Cholangitis (PBC) in 2018. ${ }^{1}$ We audited our PBC patients to assess management of patients with PBC, and identify areas for improvement.

Methods Patients were identified by searching PAS and the Northern Ireland Electronic Care Record (NIECR). ICD-10 code K74.3 was used - 'Chronic nonsupparative destructive cholangitis' - as primary and secondary diagnosis. We audited the notes of all patients who had a diagnosis of PBC in our hospital catchment area within the last 10 years based on recommendations from the 2018 BSG PBC guidelines.

Results A total of 51 patients with PBC were identified. Mean age 66 [age range 31 to 92], 11 male and 40 female [78\% F; $22 \% \mathrm{M}]$.

Of our patients, $84 \%$ were on Ursodeoxycholic Acid (UDCA), but only 54\% were on the correct dose of $13-15 \mathrm{mg} /$ $\mathrm{kg} /$ day of UCDA.

6 patients considered high risk on their LFT results has not been referred for second line therapy. 3 UDCA intolerant patients had not been referred for second line therapy.

98\% (50/51) had had baseline TFTs checked; 51\% (26/51) had had coeliac serology checked. Bone densitometry (DEXA scan) had been performed on $63 \%$ of our patients with PBC.

14 patients reviewed during our audit had passed away over the last 10 years. Of these 50\% (7/14) had died of PBC or a liver related illness.

Conclusions The majority of patients were on UDCA, but not all were on the correct dose. Of concern were the high risk patients and UDCA-intolerant patients who had not been referred onwards for consideration of second line therapy and consideration of liver transplant. COVID19 lockdown certainly negatively influenced the optimal management of PBC patient over the last 12 months. To improve our care, we have instituted a PBC proforma, to be used during clinics and aim to streamline all $\mathrm{PBC}$ patients into one specialist clinic, rather than the current multi-clinic, multi-consultant set up. The recent addition of a Fibroscan service will also benefit patient management.

\section{REFERENCE}

1. Hirschfield GM, Dyson JK, Alexander GJM et al. 2018, Gut, e-publication, 2018, page $0-27$

\section{PTH-53 EVALUATING THE EFFECT OF COVID ON AN UPPER GI TWO WEEK WAIT PATHWAY}

Amy Woods*, Sabina Beg, Anum Javed. Imperial College Healthcare Nhs Trust, London, UK

10.1136/gutjnl-2021-BSG.368

Introduction The covid-19 pandemic has necessitated a change in how healthcare provision is delivered. As such 